\title{
Development and Characterization of 68 Microsatellite Markers of Black Amur Bream Megalobrama terminalis by Next-Generation Sequencing
}

\author{
Kai Liu ${ }^{1}{ }^{(}$, Xiao-yu Feng $^{1}$, Heng-jia $\mathrm{Ma}^{1}$, Nan $\mathrm{Xie}^{2, *} \mathbb{C}$ \\ ${ }^{1}$ Hangzhou Academy of Agricultural Sciences, Institute of Fishery Science, Hangzhou, Zhejiang, China \\ How to cite
}

Liu, K., Feng, X., Ma, H., Xie, N. (2021). Development and Characterization of 68 Microsatellite Markers of Black Amur Bream Megalobrama terminalis by Next-Generation Sequencing. Turkish Journal of Fisheries and Aquatic Sciences, 21, 299-308. http://doi.org/10.4194/1303-2712v21_6_05

\section{Article History}

Received 16 November 2020

Accepted 02 March 2021

First Online 04 March 2021

\section{Corresponding Author}

Tel.: +86057187647786

E-mail: n.xie@163.com

\section{Keywords}

Megalobrama terminalis

Next-generation sequencing

Microsatellite

Polymorphism

\begin{abstract}
This study developed and characterized 68 novel polymorphic microsatellite markers from black Amur bream Megalobrama terminalis by next-generation sequencing. Variability was tested on 36 individuals collected from Qiantang River, Zhejiang Province, China. The number of alleles per locus ranged from 2 to 13. Observed heterozygosity ranged from 0.028 to 0.944 , whereas the expected heterozygosity ranged from 0.028 to 0.887 . Polymorphism Information Content ranged from 0.027 to 0.862. Moreover, 53 microsatellites were in agreement with Hardy-Weinberg equilibrium. Twenty-eight pairwise tests in 33 microsatellite loci indicated linkage disequilibrium. These microsatellites are a valuable tool for further genetics studies of this species.
\end{abstract}

\section{Introduction}

Black Amur bream Megalobrama terminalis (Richardson, 1846), a Cyprinidae family member, is a benthopelagic freshwater omnivorous fish (Froese \& Pauly, 2019). It is endemic to Asia and widely distributed from the Amur basin between the Russian Far East and Northeastern China to Southern China's rivers (Froese \& Pauly, 2019). Megalobrama terminalis is a delicious and nutritious fish. Therefore, it is regarded as a high-quality fish and deeply favored by consumers. However, in recent years, this fish's wild resources have declined due to overfishing, water pollution, and growing market demand (Hu \& Shi, 2020). At present, the Qiantang River in East China has a certain amount of resources due to developing a protective plan for local $M$. terminalis germplasm resources and has the only national original breeding farm of M. terminalis in China (Hu \& Shi, 2020).
Nevertheless, the conservation of the natural populations of this species is an urgent need.

Genetic data for this organism is scarce. Scientific studies are also necessary for the conservation of this species. Researches based on molecular markers are an essential method for estimating polymorphism and structures in wild populations. Microsatellites are often referred to as simple sequence repeats (SSRs). SSRs are highly polymorphic codominant markers and are suitable for genetic diversity assessments in fish (Chistiakov et al., 2006; Ma et al., 2011; Liu et al., 2011; Song et al., 2016; Song et al., 2017). Although twentyfour microsatellite loci excepted from markers developed by us of $M$. terminalis have been registered in GenBank, relevant information on these markers, such as primer sequences and level of polymorphisms, has not yet been published. Thus, the utility of these microsatellite loci as genetic markers remains uncertain. 
Besides, most of the microsatellite loci registered in GenBank are dinucleotide or mononucleotide repeats. The polymorphism of mono-SSRs is challenging to interpret (Lopez et al., 2015), di-SSRs are more challenging to score accurately because of substantial strand slippage during PCR (Weber et al., 2001), same as tri-SSRs. However, tetra-SSRs reduced strand slippage during PCR compared to dinucleotide repeats (Ghebranious et al., 2003). Moreover, penta-SSRs may have lower stutter proportions than tetranucleotides (De Barba et al., 2017). Meanwhile, a few microsatellite markers developed from $M$. amblycephala, $M$. hoffmanni, and $M$. pellegrini are typically used on $M$. terminalis for genetic diversity evaluation (Zhang et al., 2014; Du et al., 2016; Song et al., 2016; Song et al., 2017). Zhang et al. (2014) reported 18 out of 60 polymorphic microsatellite markers from the genomic DNA and transcriptome of M. amblycephala could be successfully amplified on M. terminalis. Du et al. (2016) found that 13 out of 30 microsatellite markers from the M. amblycephala transcriptome database could be successfully amplified on $M$. terminalis, and 11 out of 13 loci are polymorphic. Five out of 29 polymorphic microsatellite markers from the genomic DNA of $M$. pellegrini could be successfully amplified on $M$. terminalis (Song et al., 2016). Thirty out of 37 microsatellite markers from the genomic DNA of $M$. hoffmanni could be successfully amplified on $M$. terminalis, but only 4 out of 30 loci are polymorphic (Song et al., 2017). In addition, most of the microsatellite markers identified (Zhang et al., 2014; Du et al., 2016) are dinucleotide repeats, which are more difficult to score specifically because of significant strand slippage during the PCR (Weber et al., 2001). Thence, in this study, microsatellite loci were targeted to tetra-, penta-, or hexa-SSRs.

Compared with traditional microsatellite marker development methods, next-generation sequencing (NGS) is more cost-efficient (Zheng et al., 2013; Liu et al., 2017). Microsatellite markers derived from expressed sequence tags (ESTs) have achieved high efficiency in gene mapping by EST-simple sequence repeats (ESTSSRs). EST-SSRs are associated with the recognized feature genes and a useful tool for studying a fish population's genetic structure (Gao et al., 2012; Hasselman et al., 2013). Twenty-four microsatellite loci excepted from markers developed by us of $M$. terminalis have been registered in GenBank. However, molecular markers for $M$. terminalis are still not enough, which cannot fully evaluate the germplasm genetics and molecular-assisted breeding system of this species. For example, Bouza et al. (2012) constructed a consensus gene-enriched genetic map of the turbot based on 463 SNP and microsatellite markers in nine reference families, and Feng et al. (2018) used 7,820 SNPs and 295 SSRs to construct the common carp's high-density linkage map. Therefore, more molecular markers for this fish urgently need to be developed.

\section{Materials and Methods}

\section{Sample Collection and Genomic DNA Extraction}

M. terminalis utilized in this study were randomly harvested from the national original breeding farm of black Amur bream from Qiantang River, Zhejiang province, China. Fin clips samples were used, obtained, and stored in anhydrous alcohol at $-20^{\circ} \mathrm{C}$ before DNA was extracted. Genomic DNA derived by M. terminalis was achieved following a standard phenol-chloroform extraction method (Green \& Sambrook, 2012).

\section{RNA Extraction and Sequencing}

Transcriptomes of liver tissue were sequenced to access many diverse transcripts because the liver is a highly complex organ with a complex transcriptome (Shackel et al., 2002; Shackel et al., 2006). The livers of 6 $M$. terminalis were collected and mixed, used as a sequence sample to build a cDNA library. The sample tissues were frozen in liquid nitrogen and stored at $-80^{\circ} \mathrm{C}$ until use. The PCR products were sent to Personal Gene Technology CO., Ltd (Shanghai, China) for HiSeq ${ }^{\mathrm{TM}}$ 2000 sequencing.

\section{EST-SSR Detection and Primer Development}

Microsatellites within the unigenes assembly were detected using a Perl script MISA (http://pgrc.ipkgatersleben.de/misa/misa.html). The SSR loci were considered to target microsatellites with at least five tandem repeats for tetra-, penta -, and hexa-SSRs. ESTSSRs primers were designed using Primer3 ver. 2.3.6 (http://sourceforge.net/projects/primer3) under the following criteria, primers' length is approximately 20 bp. The melting temperature was around $60^{\circ} \mathrm{C}$.

\section{PCR Conditions and Amplification of Microsatellites}

Genomic DNAs from $3 \mathrm{M}$. terminalis individuals were used to initially screening primers that TouchDown PCR can amplify. According to the manufacturer's instructions, PCR amplifications were conducted using AmpliTaq Gold ${ }^{\mathrm{TM}}$ Fast PCR Master Mix (Applied Biosystems, Foster City, CA, USA). PCR amplifications were conducted at the following conditions: initial denaturation for $3 \mathrm{~min}$ at $94^{\circ} \mathrm{C}$, followed by ten cycles for $30 \mathrm{~s}$ at $94^{\circ} \mathrm{C}, 30 \mathrm{~s}$ at the annealing temperature, and $20 \mathrm{~s}$ at $72^{\circ} \mathrm{C}$; the initial annealing temperature was $65^{\circ} \mathrm{C}$, which reduced by $1^{\circ} \mathrm{C}$ in each cycle, followed by 20 cycles for $30 \mathrm{~s}$ at $94^{\circ} \mathrm{C}, 30 \mathrm{~s}$ at $55^{\circ} \mathrm{C}$, and $20 \mathrm{~s}$ at $72^{\circ} \mathrm{C}$; that was followed by a final extension at $72^{\circ} \mathrm{C}$ for $5 \mathrm{~min}$. Amplification products were analyzed by $3 \%$ agarose gel electrophoresis. The loci which cannot be amplified in all samples were excluded from further testing. All primers showed stable PCR results were labeled with a fluorescent dye (FAM) on each forward primer. 


\section{Genotyping}

Microsatellite loci were characterized by 36 individuals of $M$. terminalis randomly harvested from the national original breeding farm of black Amur bream from Qiantang River, Zhejiang province, China. PCR amplifications were conducted as same as the procedure described above. PCR amplifications were also carried out with the related parameters described above, while the final extension for PCR was conducted at $72^{\circ} \mathrm{C}$ for $30 \mathrm{~min}$. The PCR products were sequenced on an $A B I 3730 x \mid$ automated sequencer (Applied Biosystems, Foster City, CA, USA). The fragment size of alleles was determined against the standard size of Liz500 using GeneMapper software (Applied Biosystems, Foster City, CA, USA).

\section{Microsatellite Data Analysis}

The program Cervus ver. 3.0.7 assessed the number of alleles per locus, observed heterozygosity, expected heterozygosity, and the polymorphism information content (PIC; Kalinowski et al., 2007). The program PopGene ver. 1.32 assessed confirmation of Hardy-Weinberg equilibrium with the likelihood ratio test (Yeh et al., 1999). The program Arlequin ver. 3.5.2.2 carried out pairwise tests for linkage disequilibrium (Excoffier \& Lischer, 2010), performed using a likelihood ratio test whose empirical distribution was obtained by a permutation procedure (Slatkin \& Excoffier, 1996). Meanwhile, Bonferroni corrections were used to evaluate the significance (Rice, 1989). The program Micro-Checker ver. 2.2.3 (Van Oosterhout et al., 2004) was used to calculate the null allele frequency.

\section{Results and Discussion}

The Gene Ontology (GO) is a controlled vocabulary composed of $>38000$ precise defined phrases called GO terms that describe the molecular actions of gene products, the biological processes in which those actions occur, and the cellular locations where they are present (Balakrishnan et al., 2013). GO annotation is the statement of a connection between a type of gene product and the types designated by terms in an ontology (Hill et al., 2008). In this study, 68 microsatellite-containing unigenes were classified by their biological process, molecular function, and cellular component using the Blast2GO program (https://www.blast2go.com/) and visualized by the WEGO program (http://wego.genomics.org.cn/). Among the 68 unigenes, 20 were successfully mapped with $\mathrm{GO}$ annotations and classified into three ontologies that contained $23 \mathrm{GO}$ terms (Figure 1). At the cellular GO level, there were nine total GO terms, corresponding to 3 unigenes in the cell part (GO: 0044464), three unigenes in the cell (GO: 0005623), and three unigenes in the membrane (GO: 0016020); Regarding the

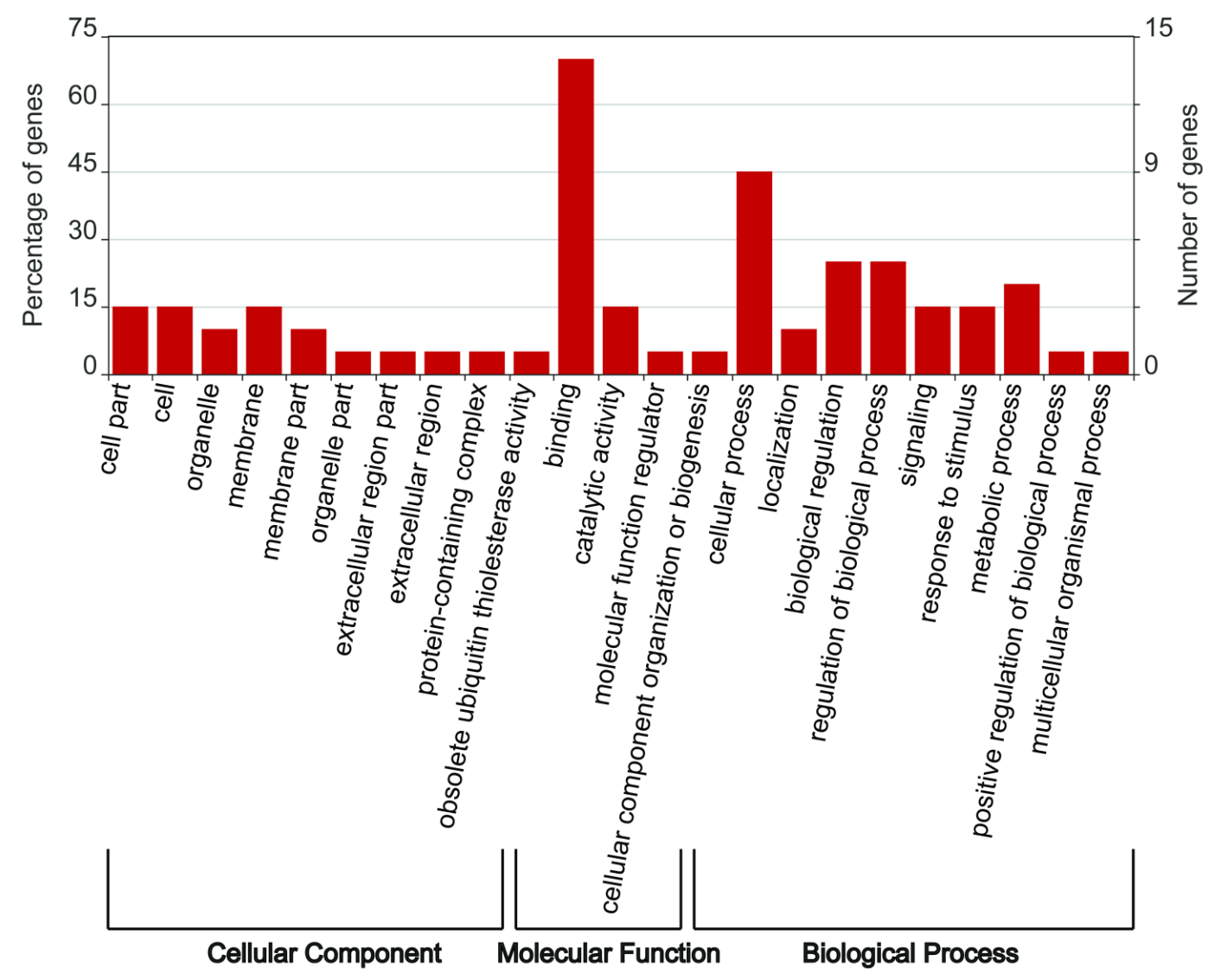

Figure 1. Gene ontology classification of 68 unigenes containing microsatellites in Megalobrama terminalis 
molecular function ontology, four total GO terms were assigned, and the primary functions were binding functions (GO: 0005488) with 14 unigenes, and catalytic activity (GO: 0003824) with three unigenes. In the biological process category, $10 \mathrm{GO}$ terms were assigned; most of the unigenes were involved in the cellular process (GO: 0009987), biological regulation (GO: 0065007), and regulation of biological process (GO: 0050789).

Sixty-eight unigenes produced by RNA-seq data contained 72 microsatellites with at least five tandem repeats for tetra-, penta-, and hexa-SSRs. There are four unigenes, and each unigenes contains two microsatellite loci. Four loci of the four unigenes were not capable of designing primers. In the 72 microsatellites, Twenty-four motifs were obtained, of which the most frequent was AAAC/GTTT (11, 15.28\%), followed by AAAG/CTTT (10, 13.89\%), AGAT/ATCT (10, 13.89\%), AAAT/ATTT (6, 8.33\%), AATC/ATTG $(6,8.33 \%)$, AAAAG/CTTTT $(4,4.17 \%)$ and AAAAT/ATTTT (4, 4.17\%) (Figure 2). Detailed analysis showed that tetra-SSRs were the most frequent (79.17\%), followed by penta-SSRs (15.28\%) and hexaSSRs $(5.55 \%)$. SSRs with five tandem repeats (27, $37.50 \%$ ) were the most common, followed by six tandem repeats $(18,25.00 \%)$ and seven tandem repeats $(9,12.50 \%)$.
All 68 microsatellite loci had a polymorphic pattern (Table 1). The number of alleles per locus ranged from 2 (Mt01650, Mt02653, Mt03365, Mt05046) to 13 (Mt01191, Mt07858). The observed heterozygosity ranged from 0.028 (Mt01650, Mt02088) to 0.944 (Mt06235), whereas the expected heterozygosity ranged from 0.028 (Mt01650) to 0.887 (Mt01210). PIC ranged from 0.027 (Mt01650) to 0.862 (Mt01210) with 42 out of which being highly informative $(P I C>0.5)$ and 20 moderately informative $(0.25<P I C<0.5)$ (Botstein et al., 1980). Analysis with the program Micro-Checker showed, in a pool of sampled individuals, low-tomoderate frequencies of null alleles in these loci, which is not surprising. Null alleles are commonly observed in various species (Zhao et al., 2011; Yu et al., 2019). Null frequencies below 0.2 are acceptable in most microsatellite datasets (Dakin \& Avise, 2004). In 14 out of 68 microsatellite loci, this estimate was higher than 0.2. According to the analyzed result of Micro-Checker, in nine out of the 14 microsatellite loci, this estimate was higher due to the stutter peak's existence. In the other five out of 14 loci, the most probable reason for this phenomenon being scoring errors may be the heterozygote genotype's loss. Fifteen out of 68 microsatellite loci exhibited significant probabilities of departure from Hardy-Weinberg equilibrium

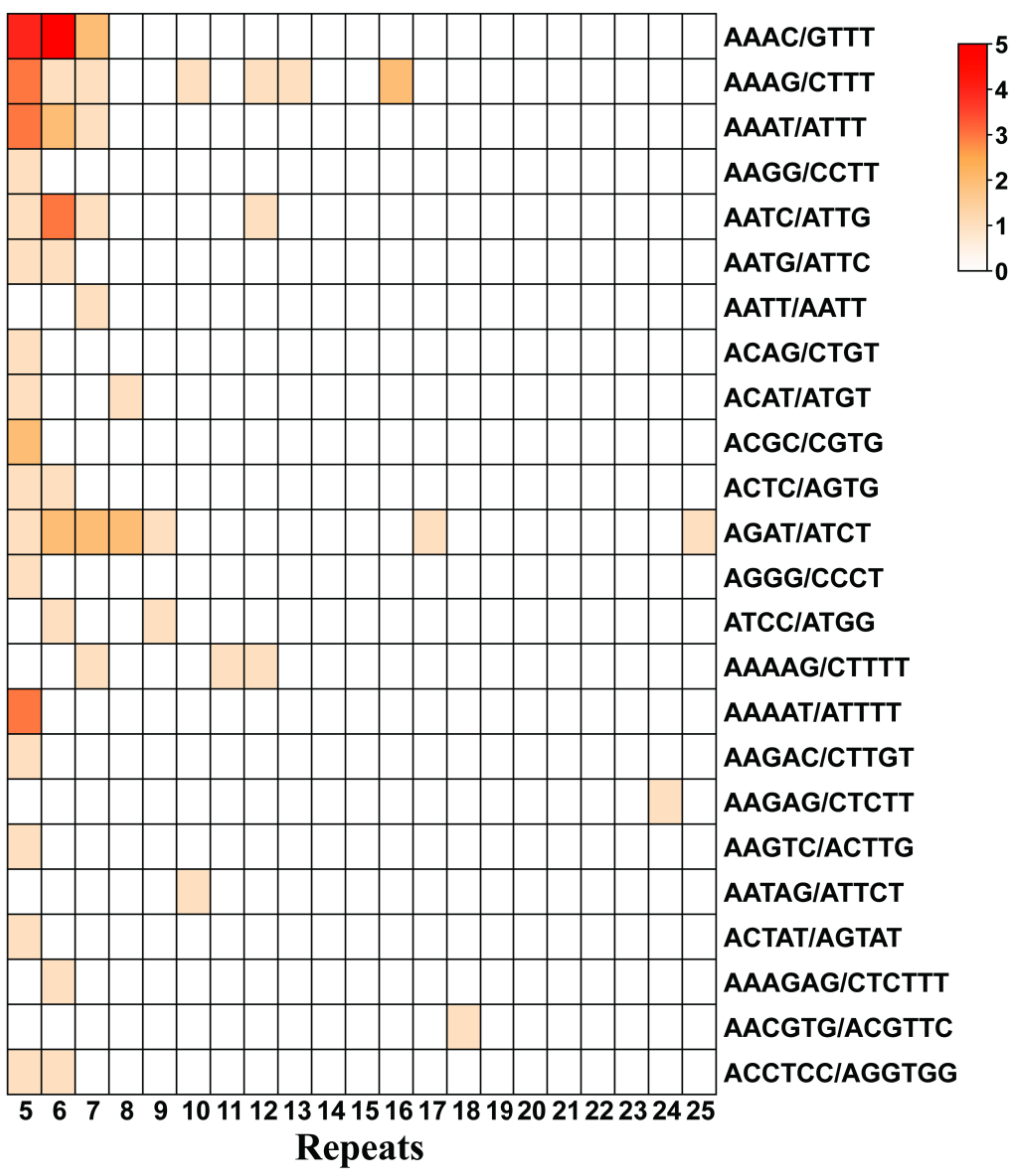

Figure 2. classification of tetra-, penta-, and hexa-SSRs of 68 unigenes in Megalobrama terminalis (considering sequence complementary) 
Table 1. Characteristics and genetic diversity of 68 microsatellite loci developed in Megalobrama terminalis

\begin{tabular}{|c|c|c|c|c|c|c|c|c|c|c|c|}
\hline ID & Repeat motif & Forward Primer(5'-3') & Reverse Primer(5'-3') & Size range & $\mathrm{Na}$ & Ho & $\mathrm{He}$ & $P I C$ & PHW & $F_{\text {Null }}$ & Accession No. \\
\hline Mt00453 & (TAGTA)5 & CCACCAGTGTGAACGCTAAA & TGCTATCCCAAAGAAAACCG & $185-195$ & 3 & 0.361 & 0.567 & 0.488 & 0.0083 & 0.1269 & MT182737 \\
\hline Mt00943 & (TGTT)6 & ATCCTCATCAGGGACATTGC & AGGTAGAGCAGATGGGCAAA & $171-187$ & 4 & 0.472 & 0.46 & 0.391 & 0.5824 & -0.0130 & MT182738 \\
\hline Mt00998 & (СТTTCT)6 & AGGGGGATCGAGAGACAGAT & CACAACACCCTGACAACCAC & $226-250$ & 5 & 0.514 & 0.636 & 0.567 & 0.0396 & 0.0984 & MT182739 \\
\hline Mt01091 & $(\mathrm{AAAC}) 6$ & TGCCATATAGAACAATACATGAAGG & ACCTTATGGACAATGCAGCC & $114-130$ & 4 & 0.306 & 0.398 & 0.364 & $0.0004^{*}$ & 0.0623 & MT182740 \\
\hline Mt01146 & (TATC)5 & AGGAATCCAACCGTCACATC & ATTGTGCTGCTGCATTGAAC & $183-207$ & 7 & 0.629 & 0.765 & 0.718 & 0.6800 & 0.0909 & MT182741 \\
\hline Mt01148 & $($ GAAA) 5 & CCTAAGGGTGAGGGCTTTTC & TCTTGTCAGGAGTGTGGGTG & $130-178$ & 6 & 0.278 & 0.647 & 0.584 & $0.0000^{*}$ & 0.2200 & MT182742 \\
\hline Mt01153 & $(\mathrm{ATAG}) 8$ & TGATCCCCTTTGGTTTTCAG & CACCCCAAGTACCCTAGCAA & $263-315$ & 10 & 0.528 & 0.751 & 0.700 & 0.6176 & 0.1221 & MT182743 \\
\hline Mt01191 & $(\mathrm{AGAT}) 7$ & GTTTCACCAAAACGGGAAAA & GGAGCGAAGTGAAGACCAAG & $259-383$ & 13 & 0.382 & 0.796 & 0.763 & 0.4516 & 0.3445 & MT182744 \\
\hline Mt01210 & $(\mathrm{TCCA}) 9$ & GACTCCTTCACCTGCGTCTC & CTCACTACAGGCCCCCAATA & $204-288$ & 12 & 0.667 & 0.887 & 0.862 & 0.1412 & 0.1109 & MT182745 \\
\hline Mt01650 & (TCCACC)5 & ACATCAGGTACGGGCTCAAC & СACTCCCACCAGTCAAGGTT & $268-274$ & 2 & 0.028 & 0.028 & 0.027 & 1.0000 & -0.0004 & MT182746 \\
\hline Mt01770 & $(\mathrm{AAAG}) 5$ & TGGGCTTTTGAGGGTACAAA & ATGCGTTTCTTGGCTTGACT & $189-281$ & 7 & 0.444 & 0.720 & 0.665 & 0.0207 & 0.1552 & MT182747 \\
\hline Mt02032 & (ATCA)12 & GGCCCAGTGACCTTCAACTA & CCCCTTTAAAATCCTTGGGA & $79-115$ & 7 & 0.686 & 0.700 & 0.651 & 0.5949 & 0.0030 & MT182748 \\
\hline Mt02088 & (ATTTT)5 & GATGCCGCCAAGAACTAAAA & ATTCCAAGCAAGGCAAAGAG & $263-278$ & 4 & 0.028 & 0.184 & 0.176 & $0.0001^{*}$ & 0.1303 & MT182749 \\
\hline Mt02229 & (TATT)6(TGTT)6 & AAATTGGCGATGAGAACTGG & CAGCTGCTAGTAATTTTAAAGCTCCA & $212-276$ & 8 & 0.361 & 0.783 & 0.736 & $0.0001^{*}$ & 0.2320 & MT182750 \\
\hline Mt02292 & (СTTT) 16 & TGGTTTATTGCCTGCTTGGT & GAGCTAAAGATCAAAAACATGCTC & $213-261$ & 10 & 0.500 & 0.646 & 0.612 & 0.7871 & 0.0839 & MT182751 \\
\hline Mt02417 & (TATT)6(TGTT)6 & CGATGAGAACTGGGCAAGA & TGGACCAGAGAGAAATGATGC & $235-351$ & 12 & 0.343 & 0.719 & 0.682 & 0.2466 & 0.3481 & MT182752 \\
\hline Mt02610 & $(\mathrm{AAGA}) 6$ & AATTTAACCAACACTTTATTACCAAGA & TCCAGACCTTCATGTGTCCA & $211-223$ & 4 & 0.194 & 0.182 & 0.171 & 0.9956 & -0.0124 & MT182753 \\
\hline Mt02653 & (GATG)6 & GCCATTACTGCTGGATGGTT & ATGAGATATTGGCGTCCGTC & $270-278$ & 2 & 0.472 & 0.504 & 0.373 & 0.7053 & 0.0162 & MT182754 \\
\hline Mt02681 & $(A A G A C) 5$ & TGGGGTCCATTTAATTCCAA & TGATGGCATGAGAACGGTAA & $189-264$ & 4 & 0.389 & 0.534 & 0.415 & 0.4005 & 0.0902 & MT182755 \\
\hline Mt02770 & (TTCA)6 & ACATTTGTATCGCTCGGAGG & CTGTCGATGCCAGCTCAATA & $248-272$ & 5 & 0.528 & 0.464 & 0.416 & 0.0659 & -0.0485 & MT182756 \\
\hline Mt03071 & $(A A A G) 7$ & GGACAATTTTGATTTCAAAGGC & CCTGTAGATGGCAAGGTGGT & $202-258$ & 6 & 0.389 & 0.477 & 0.425 & 0.5713 & 0.0556 & MT182757 \\
\hline Mt03327 & (TGAT)6 & CTGCAGTGTTCCTCACATGG & TCATTCACAGTAACTGCCTTTCA & $251-259$ & 3 & 0.472 & 0.548 & 0.465 & 0.0920 & 0.0443 & MT182758 \\
\hline Mt03365 & (ATGT)8 & TTTTCTGAGGTGGGATGGAC & GATCAGAAAATCCTGCTCCG & $221-225$ & 2 & 0.444 & 0.501 & 0.372 & 0.4936 & 0.0331 & MT182759 \\
\hline Mt03456 & $($ GAAA) 13 & AGATGCCGAAAGCTTGTGAT & CAAAGATCGGAAGGCTGGTA & $239-271$ & 9 & 0.528 & 0.772 & 0.733 & 0.0695 & 0.1325 & MT182760 \\
\hline Mt03576 & (TTTG)5 & TTGCCGCCTTAAGTCAAAGT & ATCTAGACGGCATTTCGGTG & $177-205$ & 6 & 0.917 & 0.711 & 0.644 & 0.0111 & -0.1268 & MT182761 \\
\hline Mt03643 & (GAGT)5 & GGCGTGTCCTGAGTTTTGAT & ACGTACATTCAGGGCGTCTC & $89-101$ & 4 & 0.833 & 0.632 & 0.578 & $0.0003 *$ & -0.1295 & MT182762 \\
\hline Mt03873 & (TGACT)5 & GGCTGCTTTGACACAATCTG & ATCGCATATCGTTACAGCCC & $197-262$ & 8 & 0.361 & 0.642 & 0.589 & 0.1485 & 0.1667 & MT182763 \\
\hline Mt04004 & (AAAC) 5 & GCTTGCTTATGTCCACACCA & TGAATTTTGTGAGGGGTCAA & $251-267$ & 3 & 0.222 & 0.286 & 0.249 & 0.5038 & 0.0466 & MT182764 \\
\hline Mt04221 & (TTTTC)12 & TGGCTGCAGATGAAATTAAGG & GGTTTGCACAAGTTGGTGTG & $218-278$ & 10 & 0.306 & 0.838 & 0.805 & $0.0002 *$ & 0.2852 & MT182765 \\
\hline Mt04243 & (AAAT) 5 & TACAATGCGATAGTGCTGCC & GCCGCCAGATATTCTTCAAA & $213-225$ & 4 & 0.306 & 0.435 & 0.396 & 0.0122 & 0.0861 & MT182766 \\
\hline Mt04398 & (TTTTC)7 & AGATTCTGGCTGCAGATGAAA & GGTTTGCACAAGTTGGTGTG & $224-289$ & 8 & 0.194 & 0.747 & 0.698 & $0.0000^{*}$ & 0.3123 & MT182767 \\
\hline Mt04429 & $($ TAGA) 6 & GCACTGGCAGAAATCCAAGT & ATTCTGAGCACCAGATGGGT & $179-195$ & 5 & 0.694 & 0.684 & 0.616 & 0.0204 & -0.0120 & MT182768 \\
\hline Mt04680 & (CAAA)7 & GCGTCAGAAGAGAAGGACTGA & TCAGACGCTTGTGAGGAATG & $207-275$ & 8 & 0.750 & 0.789 & 0.744 & 0.0638 & 0.0156 & MT182769 \\
\hline Mt04878 & $(\mathrm{ACGC}) 5$ & TTACTCCAGAGCACACGCAC & GGCAGTCATGCACTTTCTGA & $100-108$ & 3 & 0.333 & 0.502 & 0.397 & 0.0041 & 0.1084 & MT182770 \\
\hline Mt04992 & (TAGA) 6 & GCAGCCTTGGTGAGCATAAG & GCAGTGGATACATCAGACAGGA & $188-220$ & 6 & 0.417 & 0.668 & 0.621 & $0.0000^{*}$ & 0.1458 & MT182771 \\
\hline Mt05046 & (TCAA) 5 & AGCTGTAGCCGAGATCCTCA & СTCTCACATGTTTGTGCGGT & $192-200$ & 2 & 0.500 & 0.505 & 0.374 & 0.9474 & -0.0010 & MT182772 \\
\hline Mt05103 & (ССТC)5 & TGTTATCGGTTCTCCTCAGCA & CTTTGAACGTGTCTGCGGTA & $202-246$ & 6 & 0.611 & 0.696 & 0.623 & 0.8766 & 0.0444 & MT182773 \\
\hline Mt05108 & (TTTC)5 & GCAGGTATGCACCCCTGATA & CTCAATAAGCAGCCCAAAGG & $187-203$ & 5 & 0.444 & 0.554 & 0.449 & 0.1837 & 0.0661 & MT182774 \\
\hline
\end{tabular}


Table 1. Continued

\begin{tabular}{|c|c|c|c|c|c|c|c|c|c|c|c|}
\hline ID & Repeat motif & Forward Primer(5'-3') & Reverse Primer(5'-3') & Size range & $\mathrm{Na}$ & Ho & $\mathrm{He}$ & $P I C$ & PHW & $F_{\text {Null }}$ & Accession No. \\
\hline Mt05212 & (GATT)7 & AAACCATCTTCATCAACCGC & CGAGCCAAACTTACCTGTCC & $112-132$ & 5 & 0.722 & 0.584 & 0.525 & 0.0012 & -0.0930 & MT182775 \\
\hline Mt05488 & (GTGA)6 & CGAAGACTCGTTCTGGTTGC & GAAAAACTGCCAGGGAAACA & $219-243$ & 7 & 0.639 & 0.737 & 0.683 & 0.7312 & 0.0512 & MT182776 \\
\hline Mt05531 & (СTTC)5 & ACTACAGTACCTGCCGCTCC & CTGAGCATCATATACAGAGGCA & $266-298$ & 7 & 0.611 & 0.664 & 0.631 & 0.5160 & 0.0263 & MT182777 \\
\hline Mt05575 & (TTTC)10 & TGGTCCCCAGGACATAATTT & CCTGCTGGTCAACAGAAAGA & $254-278$ & 5 & 0.917 & 0.777 & 0.726 & 0.0753 & -0.0850 & MT182778 \\
\hline Mt05612 & (TATT)7 & AAATTGGCGATGAGAACTGG & TGCAAAATGTGATAATTCACGA & $248-280$ & 9 & 0.389 & 0.805 & 0.768 & 0.0013 & 0.2258 & MT182779 \\
\hline Mt05670 & (AAAG)12 & TTGGTAGTAAACTGCCATTTATTCAG & CCTGTAGATGGCAAGGTGGT & 139-175 & 4 & 0.361 & 0.466 & 0.403 & 0.4236 & 0.0674 & MT182780 \\
\hline Mt05830 & $($ TAGA) 8 & GCATTTCCCAAAGAAGAGCA & AGTGTTTATGGCCGTTTTCG & $112-152$ & 11 & 0.806 & 0.828 & 0.793 & 0.3704 & 0.0062 & MT182781 \\
\hline Mt06059 & (TTTG)5 & GCAGGTCCAAATCTGTCCAT & CGCTTTGACACCACTTTTTG & 153-169 & 5 & 0.806 & 0.685 & 0.616 & 0.0171 & -0.0778 & MT182782 \\
\hline Mt06205 & (TTCA) 5 & CTGCAGCGTTGTAATGGAGA & CAAGCTTAGCCCACAGACCT & $127-135$ & 3 & 0.889 & 0.598 & 0.517 & $0.0000 *$ & -0.1881 & MT182783 \\
\hline Mt06235 & $($ AGAAG)24 & CTTCAACATGAAGCACGCAT & GCAGGAGAGGCAGAAACAAC & $174-229$ & 10 & 0.944 & 0.806 & 0.768 & 0.5499 & -0.0836 & MT182784 \\
\hline Mt06290 & (GTCT)5 & CAATCACGCCTCTCTTCTCC & ATGCTCTTCTTGGGACGAAA & $232-240$ & 3 & 0.611 & 0.579 & 0.478 & 0.0152 & -0.0253 & MT182785 \\
\hline Mt06545 & $($ AAAAT) 5 & CCTGAAACTACTTGACCGGC & AATTTACAAAAAGCGCCGAG & $224-234$ & 3 & 0.278 & 0.246 & 0.219 & 0.6919 & -0.0283 & MT182786 \\
\hline Mt07071 & (ATTTT) 5 & AAAGTCTGCCCTAACTATCCTCC & GATACATATGCAGGGTGGGG & $217-232$ & 3 & 0.056 & 0.055 & 0.054 & 0.9987 & -0.0011 & MT182787 \\
\hline Mt07184 & (GTTT)5 & TGGCAGGAAGTTGGTTCTTT & GTTGTTGAAGCCCCCAAGTA & $225-245$ & 4 & 0.278 & 0.446 & 0.365 & 0.1043 & 0.1128 & MT182788 \\
\hline Mt07497 & (TTTTC)11 & TCCTTTGGGGAGAGAGGAGT & TGCAAGGATAGGGGTGCTAT & $179-236$ & 10 & 0.528 & 0.814 & 0.776 & 0.0037 & 0.1526 & MT182789 \\
\hline Mt07649 & $($ TAGA) 25 & AACACGAGCAGAGCATCAGA & TTTTGGCAGATTGATTTCCAC & $171-227$ & 8 & 0.389 & 0.806 & 0.767 & $0.0004 *$ & 0.2260 & MT182790 \\
\hline Mt07858 & $(\mathrm{AGAT}) 9$ & TGCAGCCTTTACATGCTAGTG & CAAGCTGCTCCTTCTTGTGA & $170-238$ & 13 & 0.444 & 0.835 & 0.805 & 0.1819 & 0.2080 & MT182791 \\
\hline Mt08470 & (TTTA)5 & GACCTGGATGCTGAACACCT & GGGGTGACTGAACTAATGTAGCA & $210-246$ & 5 & 0.444 & 0.723 & 0.665 & $0.0000 *$ & 0.1566 & MT182792 \\
\hline Mt08751 & (TTGT)7 & TGTCCATCCATACATCCCCT & ATAACACATCGCTTCCCTGG & $150-174$ & 4 & 0.556 & 0.531 & 0.413 & 0.8570 & -0.0210 & MT182793 \\
\hline Mt08967 & (TTAA)7 & TTGAGCAGAGTTTCAGTCTGTTTC & TTTCCCTGACCCTGTCAATC & $150-238$ & 7 & 0.278 & 0.642 & 0.594 & $0.0006 *$ & 0.2174 & MT182794 \\
\hline Mt08993 & (TATG)5 & TGCCTGTTCTTCAGGTTTCA & GGAAATAATGCACTTGGACACA & $140-168$ & 7 & 0.167 & 0.423 & 0.395 & 0.0077 & 0.1769 & MT182795 \\
\hline Mt09110 & (CAAA)6 & ATGGCACCCACTTTGACATT & ACCCTGCACATTTTGACACA & $221-249$ & 7 & 0.222 & 0.685 & 0.621 & $0.0001 *$ & 0.2706 & MT182796 \\
\hline Mt09258 & $($ ACGC)5 & TCAGATTCTTGGGCGTTTTC & CCCATTTCTGTGTGCAAATG & $241-281$ & 7 & 0.500 & 0.790 & 0.748 & 0.0008 & 0.1570 & MT182797 \\
\hline Mt09745 & $($ GGTGGA)6 & GGCCGTCTTTGGTATGTGTT & ACATCAGGTACGGGCTCAAC & $163-223$ & 6 & 0.361 & 0.665 & 0.596 & 0.0032 & 0.1780 & MT182798 \\
\hline Mt09943 & $(A A A G) 16$ & CTTTGAAGCTGGTATGGCGT & ATTAGCAATGCCTCCTCCCT & $216-256$ & 8 & 0.250 & 0.766 & 0.728 & $0.0000 *$ & 0.2881 & MT182799 \\
\hline Mt09962 & (AATAG)10 & CAAACGACGTATTTGTACTGCG & TGGGTGCGAAGTGTTATTATAGA & $130-275$ & 8 & 0.444 & 0.523 & 0.495 & 0.4547 & 0.0468 & MT182800 \\
\hline Mt10251 & (TATC)17 & CAAGCAATGCTCTCACAACC & TCTGTGTGGATGCTAGGGTG & $188-248$ & 6 & 0.139 & 0.301 & 0.284 & 0.0311 & 0.1217 & MT182801 \\
\hline Mt10425 & (CGTTCA)18 & TAACCTGCTGTCGGTGAGTG & AATGCGGGACATTTTCTCAA & $185-239$ & 7 & 0.139 & 0.741 & 0.698 & $0.0000 *$ & 0.3418 & MT182802 \\
\hline Mt10537 & (AATA)5 & TGACATCTTCACCCATCCAA & GCCTCCATTTCATATTTTCAAGA & $233-265$ & 7 & 0.806 & 0.693 & 0.630 & 0.0329 & -0.0724 & MT182803 \\
\hline Mt10953 & (AGAT)7 & CGACATGAGCCTCAATTGTTT & CTTTGGAATAACGGCTTGGA & $229-285$ & 10 & 0.417 & 0.820 & 0.786 & 0.0050 & 0.2166 & MT182804 \\
\hline
\end{tabular}

significant deviation after Bonferroni correction $(P<0.0007)$, PIC indicates polymorphism information content, $F_{\text {Null }}$ indicates null allele frequency 
expectations after Bonferroni correction, adjusted critical $P<0.0007$. The deviations occurred, perhaps resulting from the presence of null alleles or small sample size, or considering the potential for extensive gene flow in this species, the Wahlund effect (Johnson \& Black, 1984). Of the 2278 pairwise tests on the 68 microsatellites, 28 pairwise tests indicated linkage disequilibrium, adjusted critical $P<0.0007$ (Figure 3). The linkage disequilibrium patterns may affect genomewide association studies' success and the genomic selection and provide key information about demographic history (Yoshida et al., 2019). In this study, 28 pairwise tests in 33 microsatellite loci (Mt01148, Mt01210, Mt01770, Mt02088, Mt02229, Mt02292, Mt02610, Mt02681, Mt03071, Mt03327, Mt03456, Mt04221, Mt04398, Mt04680, Mt04878, Mt04992, Mt05575, Mt05612, Mt06059, Mt07184, Mt07497, Mt07649, Mt07858, Mt08470, Mt08751, Mt09110, Mt09745, Mt09943, Mt09962, Mt10251, Mt10425, Mt10537, Mt10953) indicated linkage disequilibrium among loci, which might be due to natural selection.

Using the fast isolation by AFLP of sequences containing repeats (FIASCO) method, we have developed 15 microsatellite markers from the genomic DNA of M. terminalis (Liu et al., 2020). However, most of the markers developed by the FIASCO method are di-
SSRs. Besides, most of the polymorphic SSRs reported by Zhang et al. (2014) are di-SSRs, too. Furthermore, 11 out of 30 polymorphic SSRs reported by Du et al. (2016) are almost di-SSRs. di-SSRs are more challenging to score accurately because of substantial strand slippage during PCR (Weber et al., 2001). Song et al. $(2016,2017)$ had developed 5 out of 29 and 4 out of 37 polymorphic tetra, penta-, and hexa-SSRs from the genomic DNA of $M$. pellegrini and $M$. hoffmanni, respectively, that could be successfully amplified on M. terminalis. However, only 9 SSRs are insufficient to evaluate germplasm genetics, especially constructing a microsatellites-based linkage map. In contrast, 68 polymorphic microsatellite markers have been developed in this research. These markers significantly increased the number of microsatellite markers of $M$. terminalis.

The SSR markers can be classified into two groups, EST-SSRs and the genomic SSRs (gSSRs). In general, the frequency of EST-SSRs can be confirmed to be decreased because SSRs have a high degree of mutation and can influence gene expression (Vieira et al., 2016). However, some studies indicate a predominance of the tri-SSRs and hexa-SSRs in coding regions, resulting from selection pressure against mutations that alter the reading frame (Zhang et al., 2004; Xu et al., 2013). In humans, tandem repeats have been documented to be

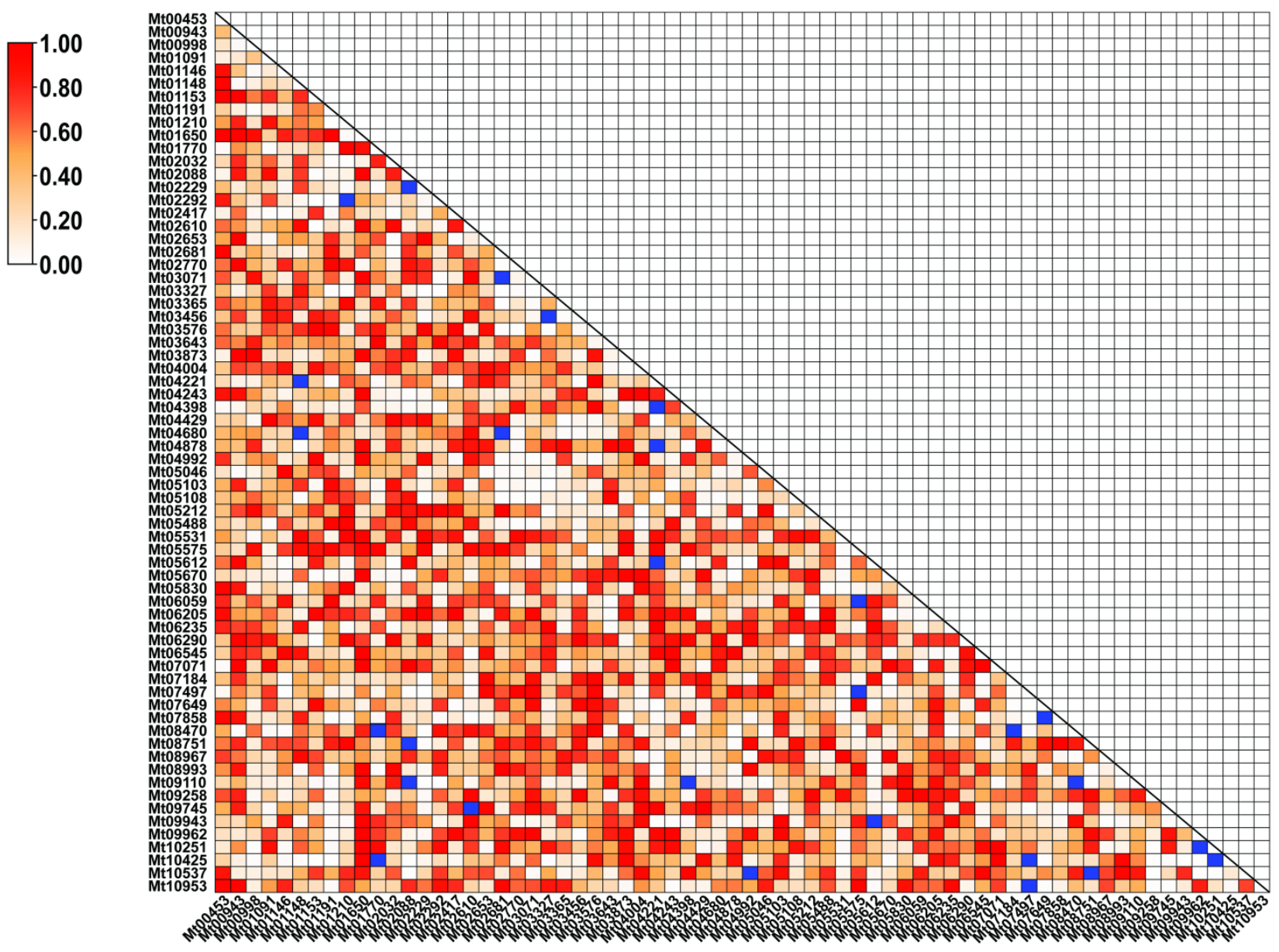

Figure 3. the probabilities from pairwise tests for linkage disequilibrium of 68 microsatellite loci in Megalobrama terminalis. Blue squares indicate a significant deviation after Bonferroni correction $(P<0.0007)$ 
expected in many proteins, and the mechanisms involved in their genesis may contribute to the rapid evolution of proteins (Vieira et al., 2016). Different SSRs have different uses. gSSRs are resulting in better map coverage, and utilization in comparative mapping and evolution studies is advantageous to EST-SSRs (Parthiban et al., 2018). The SSRs in this research are isolated within the unigenes. Therefore, such SSRs are not neutral. EST-SSRs are treated as non-neutral markers but useful for studying adaptive genetic diversity (Ellis \& Burke, 2007). Although the developed 68 SSRs are a valuable tool for further genetics studies of this species, they should be treated with caution due to the significant Hardy-Weinberg and linkage disequilibrium tests. New studies with different populations should be employed to evaluate the potential of the developed SSRs. It is expected that the reduction of the NGS cost will increase the diffusion of this approach for non-model organisms. NGS will uncover DNA polymorphisms at an unprecedented scale by making available extensive data on both gSSRs and EST-SSRs (Manco et al., 2020).

\section{Ethical Statement}

The guidelines established by the Administration of Affairs Concerning Animal Experimentation state that approval from the Science and Technology Bureau of China and the Department of Wildlife Administration is not necessary when the fish in question are neither rare nor near extinction (first- or second-class state protection level). Therefore, approval was not required for the experiments conducted in this study.

\section{Funding Information}

This study was supported by the Earmarked Fund for China Agriculture Research System under Grant CARS-45-39; Hangzhou Agricultural \& Social Development Research Program under Grant 20162012A03; Science \& Technology Innovation Program of Hangzhou Academy of Agricultural Sciences under Grant 2019HNCT-01.

\section{Author Contribution}

Kai Liu and Nan Xie conducted the experiments; Kai Liu analyzed the data and wrote the manuscript; Xiao-yu Feng and Heng-jia Ma contributed to data analysis and revising the manuscript; Nan Xie supervised the whole project.

\section{Conflict of Interest}

The authors declare that they have no known competing financial interests or personal relationships that could have influenced the work reported in this paper. The authors alone are responsible for the content and writing of the paper.

\section{Acknowledgments}

This work was supported by the Earmarked Fund for China Agriculture Research System under Grant CARS-45-39; Hangzhou Agricultural \& Social Development Research Program under Grant 20162012A03; Science \& Technology Innovation Program of Hangzhou Academy of Agricultural Sciences under Grant 2019HNCT-01. The authors declare that they have no known competing financial interests or personal relationships that could have appeared to influence the work reported in this paper. The authors alone are responsible for the content and writing of the paper.

\section{References}

Balakrishnan, R., Harris, M. A., Huntley, R., Van Auken, K., \& Cherry, J. M. (2013). A guide to best practices for Gene Ontology (GO) manual annotation. Database: the journal of biological databases and curation, 2013, bat054. https://doi.org/10.1093/database/bat054

Botstein, D., White, R. L., Skolnick, M., \& Davis, R. W. (1980). Construction of a genetic linkage map in man using restriction fragment length polymorphisms. American Journal of Human Genetics, 32, 314-331.

Bouza, C., Hermida, M., Pardo, B. G., Vera, M., Fernández, C., de la Herrán, R., Navajas-Pérez, R., Álvarez-Dios, J. A., Gómez-Tato, A., \& Martínez, P. (2012). An Expressed Sequence Tag (EST)-enriched genetic map of turbot (Scophthalmus maximus): a useful framework for comparative genomics across model and farmed teleosts. BMC genetics, 13, 54. https://doi.org/10.1186/1471-2156-13-54

Chistiakov, D. A., Hellemans, B. \& Volckaert, F. A. M. (2006). Microsatellites and their genomic distribution, evolution, function and applications: a review with special reference to fish genetics. Aquaculture, 255, 129. https://doi.org/10.1016/j.aquaculture.2005.11.031

Du, R., Zhang, D. L., Wang, Y. Z., Wang, W. M., \& Gao, Z. X. (2016). Cross-species amplification of microsatellites in genera Megalobrama and Parabramis. Journal of Genetics, 93(Suppl 2), 106-109. https://doi.org/10.1007/s12041-013-0308-1

Dakin, E. E., \& Avise, J. C. (2004). Microsatellite null alleles in parentage analysis. Heredity 93, 504-509. https://doi.org/10.1038/sj.hdy.6800545

De Barba, M., Miquel, C., Lobreaux, S., Quenette, P. Y., Swenson, J. E., \& Taberlet, P. (2017). High-throughput microsatellite genotyping in ecology: improved accuracy, efficiency, standardization and success with low-quantity and degraded DNA. Molecular Ecology Resources, 17(3), 492-507. https://doi.org/10.1111/1755-0998.12594

Ellis, J. R., \& Burke, J. M. (2007). EST-SSRs as a resource for population genetic analyses. Heredity, 99(2), 125-132. https://doi.org/10.1038/sj.hdy.6801001

Froese, R., \& Pauly, D. (2019). FishBase. https://www.fishbase.se/summary/Megalobramaterminalis.html.

Feng, X., Yu, X., Fu, B., Wang, X., Liu, H., Pang, M., \& Tong, J. (2018). A high-resolution genetic linkage map and QTL fine mapping for growth-related traits and sex in the 
Yangtze River common carp (Cyprinus carpio haematopterus). BMC genomics, 19(1), 230. https://doi.org/10.1186/s12864-018-4613-1

Gao, Z., Luo, W., Liu, H., Zeng, C., Liu, X., Yi, S., \& Wang, W. (2012). Transcriptome analysis and SSR/SNP markers information of the blunt snout bream (Megalobrama amblycephala). PLos One, 7(8), e42637. https://doi.org/10.1371/journal.pone.0042637

Ghebranious, N., Vaske, D., Yu, A., Zhao, C. F., Marth, G., \& Weber, J. L. (2003). STRP screening sets for the human genome at $5 \mathrm{cM}$ density. BMC Genomics, 4(1), 6. https://doi.org/10.1186/1471-2164-4-6

Green, M. R., \& Sambrook, J. (2012). Molecular Cloning: A Laboratory Manual. 4th Edition. New York: Cold Spring Harbor Laboratory.

Hasselman, D. J., Ricard, D., \& Bentzen, P. (2013). Genetic diversity and differentiation in a wide ranging anadromous fish, American shad (Alosa sapidissima), is correlated with latitude. Molecular Ecology, 22, 15581573. https://doi.org/10.1111/mec.12197

Hill, D.P., Smith, B., McAndrews-Hill, M.S. \& Blake, J. A. Gene Ontology annotations: what they mean and where they come from. BMC Bioinformatics 9, S2 (2008). https://doi.org/10.1186/1471-2105-9-S5-S2

Hu, X. S., \& Shi, L. Y. (2020). A Review: Research Progress on Germplasm Resource of Black Bream (Megalobrama terminalis) in China. Chinese Journal of Fisheries, 33(3): 84-89.

Johnson, M. S., \& Black, R. (1984). The Wahlund effect and geographical scale of variation in the intertidal limpet Siphonaria sp. Marine Biology, 79, 295-302. https://doi.org/10.1007/BF00393261

Kalinowski, S. T., Taper, M. L., \& Marshall, T. C. (2007). Revising how the computer program CERVUS accommodates genotyping error increase success in paternity assignment. Molecular Ecology, 16, 1099-1106. https://doi.org/10.1111/j.1365-294x.2007.03089.x

Excoffier, L., \& Lischer, H. E. (2010). Arlequin suite ver 3.5: a new series of programs to perform population genetics analyses under Linux and Windows. Molecular ecology resources, 10(3), 564-567. https://doi.org/10.1111/j.1755-0998.2010.02847.x

Liu, B., Kuang, Y. Y., Tong, G. X., \& Yin, J. S. (2011). Analysis of genetic diversity on 9 wild stocks of Taimen (Hucho taimen) by microsatellite markers. Zoological Research, 32, 597-604. https://doi.org/10.3724/sp.j.1141.2011.06597

Liu, H. G., Yang, Z., Tang, H. Y., Gong, Y., \& Wan, L. (2017). Microsatellite development and characterization for Saurogobio dabryi Bleeker, 1871 in a Yangtze riverconnected lake, China. Journal of Genetics, 96, e1-e4. https://doi.org/10.1007/s12041-016-0733-z

Liu, K., Xie, N., Feng, X. Y., \& Ma, H. J. (2020). Characterization of 15 polymorphic microsatellite markers developed by magnetic beads enrichment in Megalobrama terminalis. Journal of Zhejiang Agricultural Sciences, 61(1), 144-146. https://doi.org/10.16178/j.issn.0528-9017.20200144

Lopez, L., Barreiro, R., Fischer, M., \& Koch, M. A. (2015). Mining microsatellite markers from public expressed sequence tags databases for the study of threatened plants. BMC Genomics, 16, 781. https://doi.org/10.1186/s12864-015-2031-1

Ma, K. Y., Feng, J. B., Xie, N., Feng, X. Y., \& Li, J. L. (2011). Microsatellite analysis of genetic variation of the oriental river prawn Macrobrachium nipponense in Qiantang
River. Zoological Research, 3, 363-370. https://doi.org/10.3724/sp.j.1141.2011.04363

Manco, R., Chiaiese, P., Basile, B., \& Corrado, G. (2020). Comparative analysis of genomic- and EST-SSRs in European plum (Prunus domestica L.): implications for the diversity analysis of polyploids. 3 Biotech, 10(12), 543. https://doi.org/10.1007/s13205-020-02513-w

Parthiban, S., Govindaraj, P., \& Senthilkumar, S. (2018). Comparison of relative efficiency of genomic SSR and EST-SSR markers in estimating genetic diversity in sugarcane. 3 Biotech, 8(3), 144. https://doi.org/10.1007/s13205-018-1172-8

Rice, W. R. (1989). Analyzing tables of statistical tests. Evolution, 43, 223-225. https://doi.org/10.1111/j.1558-5646.1989.tb04220.x

Shackel, N. A., Gorrell, M. D., \& McCaughan, G. W. (2002). Gene array analysis and the liver. Hepatology, 36(6), 1313-1325. https://doi.org/10.1053/jhep.2002.36950

Shackel, N. A., Seth, D., Haber, P. S., Gorrell, M. D., \& McCaughan, G. W. (2006). The hepatic transcriptome in human liver disease. Comparative Hepatology, 5, 6. https://doi.org/10.1186/1476-5926-5-6

Slatkin, M., \& Excoffier, L. (1996). Testing for linkage disequilibrium in genotypic data using the ExpectationMaximization algorithm. Heredity, 76 (4), 377-383. https://doi.org/10.1038/hdy.1996.55

Song, W., Zhu, D., Lv, Y., \& Wang, W. M. (2016). Development and characterization of 29 polymorphic microsatellite loci of Megalobrama pellegrini by next-generation sequencing technology and cross-species amplification in related species. PeerJ Preprints, 4, e2490v1. https://doi.org/10.7287/peerj.preprints.2490v1

Song, W., Zhu, D., Lv, Y., \& Wang, W. M. (2017). Isolation and characterization of 37 polymorphic microsatellite loci of Megalobrama hoffmanni by next-generation sequencing technology and cross-species amplification in related species. Journal of Genetics, 96, 39-45. https://doi.org/10.1007/s12041-017-0815-6

Van Oosterhout, C., Hutchinson, W. F., Wills, D. P. M., \& Shipley P. (2004). MICRO-CHECKER: software for identifying and correcting genotyping errors in microsatellite data. Molecular Ecology Resources, 4, 535538. https://doi.org/10.1111/j.1471-8286.2004.00684.x

Vieira, M. L., Santini, L., Diniz, A. L., \& Munhoz, C. (2016). Microsatellite markers: what they mean and why they are so useful. Genetics and molecular biology, 39(3), 312-328. https://doi.org/10.1590/1678-4685-GMB-2016-0027

Weber, J. L., \& Broman, K. W. (2001). Genotyping for human whole-genome scans: past, present, and future. Advances in Genetics, 42, 77-96.

https://doi.org/10.1016/s0065-2660(01)42016-5

$\mathrm{Xu}$, J., Liu, L., Xu, Y., Chen, C., Rong, T., Ali, F., Zhou, S., Wu, F., Liu, Y., Wang, J., Cao, M., \& Lu, Y. (2013). Development and characterization of simple sequence repeat markers providing genome-wide coverage and high resolution in maize. DNA research, 20(5), 497-509. https://doi.org/10.1093/dnares/dst026

Yeh, F. C., Yang, R. C., Boyle, T. B. J., Ye, Z. H., \& Mao, J. X. (1999). POPGENE 1.32, the user-friendly shareware for population genetic analysis. Edmonton: Molecular Biology and Biotechnology Center, University of Alberta. https://sites.ualberta.ca/ fyeh/popgene_download.ht $\mathrm{ml}$. 
Yoshida, G. M., Barria, A., Correa, K., Cáceres, G., Jedlicki, A., Cadiz, M. I., Lhorente, J. P., \& Yáñez, J. M. (2019). Genome-Wide Patterns of Population Structure and Linkage Disequilibrium in Farmed Nile Tilapia (Oreochromis niloticus). Frontiers in genetics, 10, 745. https://doi.org/10.3389/fgene.2019.00745

Yu, J. N., Kim, S. K., Sagong, J., Ryu, S. H., \& Chae, B. (2019). Identification of microsatellite markers and their application in yellow catfish (Pseudobagrus fulvidraco Richardson, 1846) population genetics of Korea. Journal of Genetics, 98, 2-7.

https://doi.org/10.1007/s12041-018-1047-0

Zhang, L., Yuan, D., Yu, S., Li, Z., Cao, Y., Miao, Z., Qian, H., \& Tang, K. (2004). Preference of simple sequence repeats in coding and non-coding regions of Arabidopsis thaliana. Bioinformatics (Oxford, England), 20(7), 1081-
1086. https://doi.org/10.1093/bioinformatics/bth043 Zhang, Q. Q., Chen, J., Jiang, X. Y., \& Zou, S. M. (2014). Establishment of DNA fingerprinting and analysis on genetic structure of different Parabramis and Megalobrama populations with microsatellite. Journal of Fisheries of China, 38, 15-23.

Zheng, X. F., Pan, C., Diao, Y., You, Y. N., Yang, C. Z., \& Hu, Z. L. (2013). Development of microsatellite markers by transcriptome sequencing in two species of Amorphophallus (Aracease). BMC Genomics, 14, 490. https://doi.org/10.1186/1471-2164-14-490

Zhao, Y., Ji, X. S., Zeng, Y. Q., Ding, L., Yang, P. P., \& Wang, H. (2011). Isolation of microsatellite markers for Lateolabrax japonicus and polymorphic analysis. Zoological Research, 32, 515-520.

https://doi.org/10.3724/sp.j.1141.2011.05515 